\title{
Biohydrogen production from oil palm frond juice and sewage sludge by a metabolically engineered Escherichia coli strain
}

\begin{abstract}
Biohydrogen is considered a promising and environmentally friendly energy source. Escherichia coli BW25113 hyaB hybC hycA fdoG frdc ldhA aceE has been previously engineered for elevated biohydrogen production from glucose. In this study, we show that this strain can also use biomass from oil palm frond (OPF) juice and sewage sludge as substrates. Substrate improvement was accomplished when hydrogen productivity increased 8 -fold after enzymatic treatment of the sludge with a mixture of amylase and cellulase. The OPF juice with sewage sludge provided an optimum carbon/nitrogen ratio since the yield of biohydrogen increased to 1.5 from $1.3 \mathrm{~mol} \mathrm{H} 2 / \mathrm{mol}$ glucose compared to our previous study. In this study, we also reveal that our engineered strain improved 200-fold biohydrogen productivity from biomass sources compared to the unmodified host. In conclusion, we determined that our engineered strain can use biomass as an alternative substrate for enhanced biohydrogen production.
\end{abstract}

Keyword: Biohydrogen; Escherichia coli; Oil palm frond juice; Sewage sludge 